\title{
Birth Month
}

National Cancer Institute

\section{Source}

National Cancer Institute. Birth Month. NCI Thesaurus. Code C83090.

The month in which a person was born. 